\title{
The complete guide to self sufficiency
}

John Seymour, Britain's self-sufficiency guru, could have learned a lot from cancer cells: by producing growth factors and their receptors, they lose reliance on their neighbours. Thomas Graeber and David Eisenberg now describe a method for cataloguing these autocrine loops.

The authors reasoned that if a cancer cell expresses both a ligand and its receptor, their transcription might be coupled. So they compiled a database of ligand-receptor pairs from the literature, and then mined five collections of publicly available gene-expression data from human cancers and cancer-cell lines for the expression profiles of ligand-receptor pairs. They then calculated whether there was a correlation between the expression profiles of each ligand and its receptor

This identified several autocrine loops known to occur in cancer. One of these was tumour necrosis factor (TNF) and its receptor TNFR1 in diffuse large B-cell lymphoma. Expression of this pair correlated only in tumour cells from patients who had not survived beyond 4 years

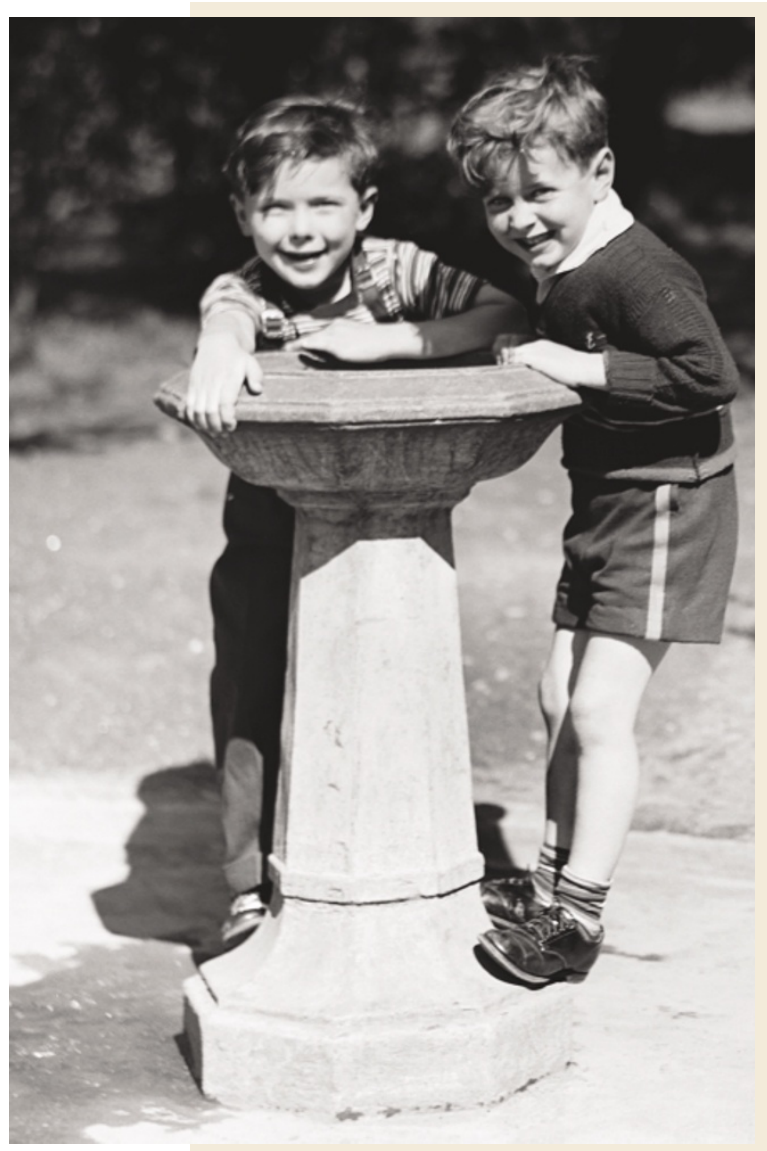

after diagnosis and treatment, illustrating the prognostic - and potential therapeutic - relevance of autocrine loops. CC chemokines and their receptors, which are involved in cell movement and might be expected to confer a metastatic phenotype, were also overrepresented.

Some pairs indicated that cancer cells might also have strategies for blocking autocrine signalling: expression of some 'decoy' receptors correlates with their ligands, and inverse correlations between ligand and receptor expression also occur. For example, in several cancer types, ERBB3 expression is downregulated whenever its ligand, neuregulin 1 , is upregulated.

This study has generated a long list of hypotheses that now need testing to determine whether the loops identified are operative. But it could provide rich pickings for the identification of prognostic markers and therapeutic targets. Might it be possible to set a pharmaceutical fox among the chickens in some of these self-sufficient systems?

Cath Brooksbank

\section{CARCINOGENESIS}

\section{Double trouble}

Ciclosporin is an effective

immunosuppressant that is widely used not only in transplant recipients, but also to treat chronic inflammatory diseases such as psoriasis. In organ transplant recipients, ciclosporin use has been associated with an increased risk of squamous-cell carcinoma, a type of skin cancer. This is a worrying observation because psoriasis is often treated with another carcinogen psoralen and ultraviolet-A light (PUVA) in combination with ciclosporin. Isabelle Marcil and Robert Stern, in the 29 September issue of The Lancet, now show that ciclosporin and PUVA are a particularly carcinogenic combination.

Marcil and Stern found that ciclosporin users had a threefold higher incidence of squamous-cell carcinoma than nonusers, whereas patients who were ciclosporin users and had experienced high PUVA exposure had a sevenfold higher incidence of squamous-cell carcinoma than ciclosporin users who had not been exposed to PUVA. After adjustment for

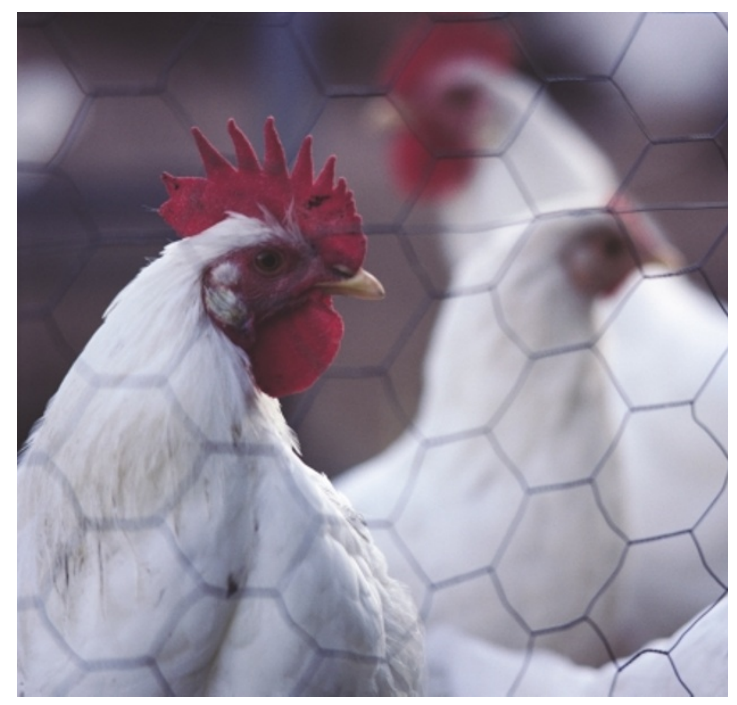

(2) References and links

ORIGINAL RESEARCH PAPER Graeber, T. G. \& Eisenberg, D.

Bioinformatic identification of potential autocrine signalling loops in cancers from gene expression profiles. Nature Genet. $\mathbf{2 9}$ 295-300 (2001)

WEB SITES

The Database of Interacting Proteins: http://dip.doembi.ucla.edu

Nature Genome Gateway - Profiling microarrays:

http://www.nature.com/genomics/post-

genomics/microarrays.html

David Eisenberg's lab: http://www.doe

mbi.ucla.edu/People/Eisenberg/

ageing (which, in itself, increases cancer incidence) during the study and use of other treatments, the incidence of squamous-cell carcinoma was ninefold higher in individuals who had received both ciclosporin and a high exposure to PUVA compared with nonusers with a low PUVA exposure. Incidence of tumours increased most in regions of the body not usually exposed to sunlight but exposed to PUVA. Perhaps most significantly, even brief or minimal use of ciclosporin greatly increases the risk of squamous-cell carcinoma in individuals who have also received long-term treatment with PUVA.

There is, therefore, a potential safety issue for patients receiving ciclosporin, and possibly also other immunosuppressants used for severe psoriasis, when they are used in conjunction with other known carcinogens. These findings indicate that further investigations into the cancercausing potential of other immunosuppressants used to treat psoriasis should be undertaken.

(4) References and links

Sandra Clark ORIGinal ReSEARCh PAPER Marcil, I. \& Stern, R. S Squamous-cell cancer of the skin in patients given PUVA and ciclosporin: nested cohort crossover study. Lancet $\mathbf{3 5 8}$ 1042-1045 (2001) 